\title{
The GAPS Instrument: A Large Area Time of Flight and High Resolution Exotic Atom Spectrometer for Cosmic Antinuclei
}

\section{S. Quinn ${ }^{a, *}$ on behalf of the GAPS Collaboration}

(a complete list of authors can be found at the end of the proceedings)

\author{
a Deptartment of Physics \& Astronomy, \\ 475 Portola Plaza, Los Angeles, USA \\ E-mail: spq@ucla.edu
}

Low-energy cosmic ray antideuterons $(<0.25 \mathrm{GeV} / n)$ are a compelling, mostly uncharted channel of many viable dark matter models and benefit from highly suppressed astrophysical background. The General Antiparticle Spectrometer (GAPS) is a first-of-its-kind exotic-atom-based Antarctic balloon-borne experiment specialized for detection of low-energy antiprotons, antideuterons, and antihelium with a targeted launch in 2022. The results of novel technology development and a summary of current construction status are the focus of this contribution.

GAPS exploits a novel antiparticle identification technique based on exotic atom formation and decay, allowing more active target material for a larger overall acceptance since no magnet is required. The GAPS instrument consists of a large-area $\left(\sim 50 \mathrm{~m}^{2}\right)$ scintillator time-of-flight, ten planes of custom silicon detectors with dedicated ASIC readout, and a novel oscillating heat pipe cooling approach.

This contribution will briefly introduce the exotic atom detection technique. Following this, the instrument design will be discussed and detailed description of experimental hardware and expected performance will be presented. I will conclude with recent construction and testing progress while also highlighting developments of a scaled, integrated prototype.

$37^{\text {th }}$ International Cosmic Ray Conference (ICRC 2021)

July 12 th - 23rd, 2021

Online - Berlin, Germany

\footnotetext{
${ }^{*}$ Presenter
} 


\section{Introduction}

GAPS is a balloon payload ( $\approx 35 \mathrm{~km}$ altitude, $5 \mathrm{~g} \mathrm{~cm}^{-2}$ overburden) designed to detect light, moderately relativistic $(\gamma \approx 1.1)$ cosmic-ray antimatter. A main objective is the contribution of new spectrum data for low kinetic energy antiprotons and flux limits antideuterons ( $\bar{p}$, and $\bar{d}$ respectively) to supplement existing experimental data at higher energies. Spectrum information $(\bar{p})$ has broad value in the cosmic ray community, e.g. for constraining propagation models, but an additional feature of the low-energy antimatter is an essentially background free channel for indirect dark matter detection-primary dark matter flux exceeds the expected background astrophysical flux by $\approx 100-300$ for multiple model candidates [1,2].

An additional objective is sensitivity to antihelium, an intrinsically challenging channel due to the minuscule level of expected flux compared to antideuterons. Based on a recent analysis reported in [3], GAPS is predicted to be sensitive to $\overline{{ }^{3} \mathrm{He}}$ produced by $m_{\chi}=100 \mathrm{GeV}$ via the $\chi \chi \rightarrow W^{+} W^{-}$ channel [4]. For a review of additional particle physics motivation for GAPS see [5, 6]. The current iteration of GAPS builds on the heritage of the successful prototype 2012 flight [7-9].

\section{Instrument design and detection technique}

The GAPS detector uses a lithium drifted silicon tracker $[\mathrm{Si}(\mathrm{Li})]$ encapsulated by a plastic scintillator time of flight system for exotic atom spectrometry instead of track bending for particle composition discrimination as used in typical magnetic spectrometers. Cosmic-ray antimatter primaries entering the instrument through a semi-vertical acceptance are degraded by active and passive material, forming an unstable exotic atom as it approaches zero kinetic energy in the target material. The exotic atom decay generates characteristic X-rays dependent on the primary species, and its annihilation produces outgoing secondary products dominated by pions and protons, see [1], which are also be detected. A diagram depicting the main features of the primaries of interest is shown in Figure 1. Since $\mathrm{Si}(\mathrm{Li})$ must be operated at colder temperatures, a novel semi-passive oscillating heat pipe solution is used for thermal management to achieve the required performance at $-40{ }^{\circ} \mathrm{C}$. Supporting data acquisition, communications and balloon-craft control electronics are grouped at the bottom of the instrument payload. For a schematic CAD view, refer to Figure 2. For more details on the detection technique and additional overviews, consult $[10,13,14]$.

\section{3. $\mathrm{Si}(\mathrm{Li})$ tracker system}

The main target is comprised of multiple layers of $\mathrm{Si}(\mathrm{Li})$ detectors used for vertex finding, particle tracking, and high-energy X-ray sensing. This section explores the individual detector elements, geometrical design, and demonstrated performance based on lab testing.

\subsection{Detector elements, geometry/mechanical, and performance}

The tracker assembly consists of a $6 \times 6$ array of modules forming a single layer. The tracker volume is a stack of 10 layers. The layers are supported and insulated by light weight foam. The thermal working fluid circulates through a latticework of capillary tubing perpendicular to the detector planes (see Figure 2) [11, 12]. 

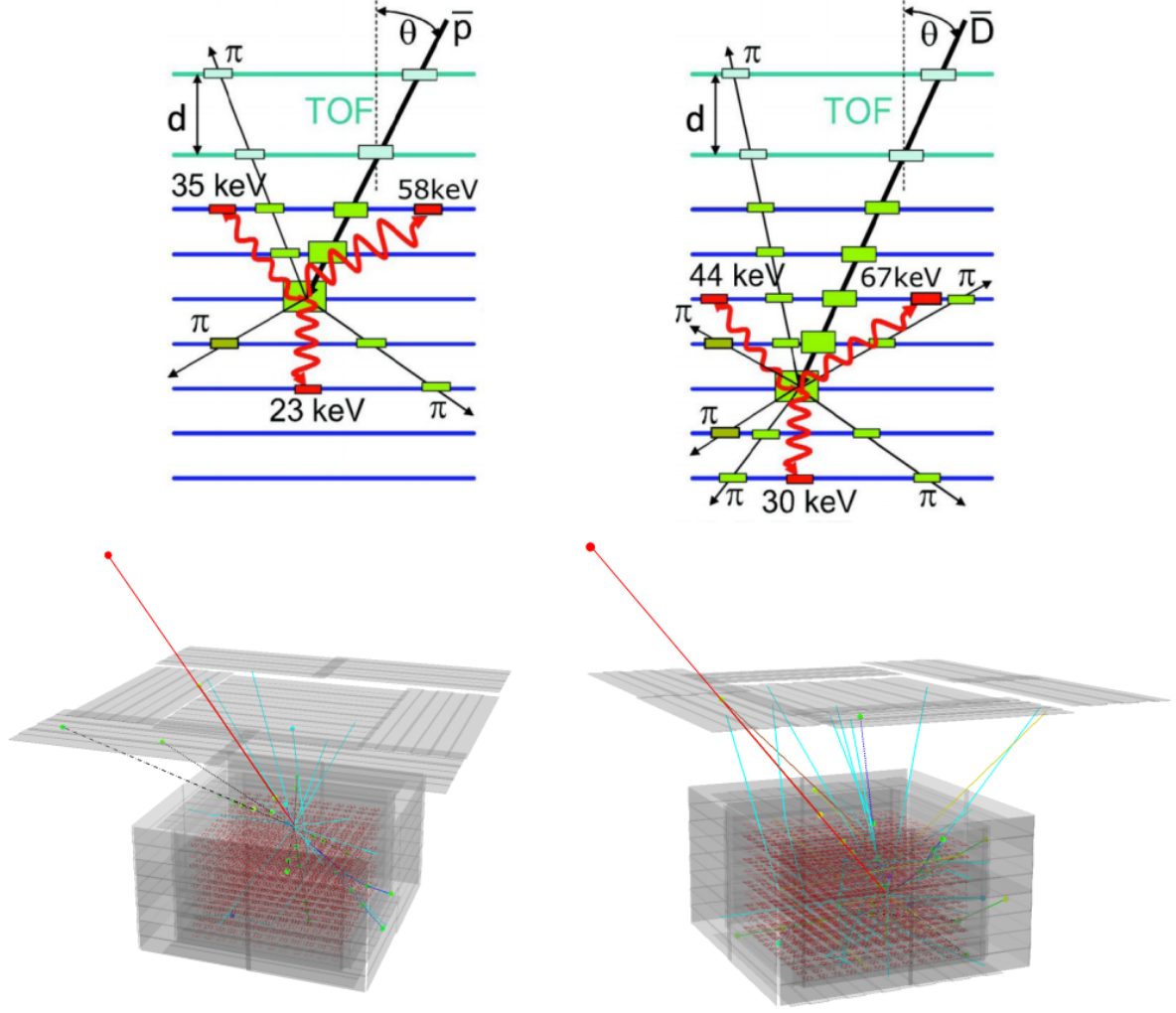

Figure 1: Top row: Cartoon of possible vertex and event topology for antiprotons (left) and antideuterons (right) [1]. Bottom: Geant4 simulation for typical events. In addition to the X-ray fingerprint, many additional features such as $\beta, \pi$-multiplicity, energy deposition profile, and primary depth can be used to discriminate between $\bar{p}$ and $\bar{d}$.

A $\mathrm{Si}(\mathrm{Li})$ detector module contains four detectors with active material of $\approx 90 \mathrm{~mm}$ diameter and $\approx 2.3 \mathrm{~mm}$ thickness. The detectors are segmented into eight equal area strips for readout. See Figure 4 for a photo of disks mounted with an unpopulated PCB. A custom passivation process was developed and validated for protecting the sensitive exposed silicon surfaces from contamination. The development, fabrication and characterization of these novel large area detectors is detailed in [15-17].

The performance of the flight-model $\mathrm{Si}(\mathrm{Li})$ detectors is well understood. At temperatures of -35 to $-45{ }^{\circ} \mathrm{C}$ expected during flight, we have achieved the $\sigma_{\mathrm{FWHM}}<4 \mathrm{keV}$ energy resolution necessary to distinguish the $20-100 \mathrm{keV} \mathrm{X}$-rays from $\bar{p}$ and $\bar{d}$ annihilation, as shown in Figure 4 [15].

\subsection{Electronics}

Using custom wire bonds, the four biased $\mathrm{Si}(\mathrm{Li})$ disks (32 channels) are connected to a fully integrated ASIC. This includes a low-noise custom analog front end and readout core carefully designed to meet challenging power $(<10 \mathrm{~mW} / \mathrm{ch})$ and density $(8,000$ total channels). Additionally, a large dynamic range ( $20 \mathrm{keV}$ to $\approx \mathrm{MeV}$ ) needed for the $\mathrm{X}$-ray physics is achieved using novel signal 


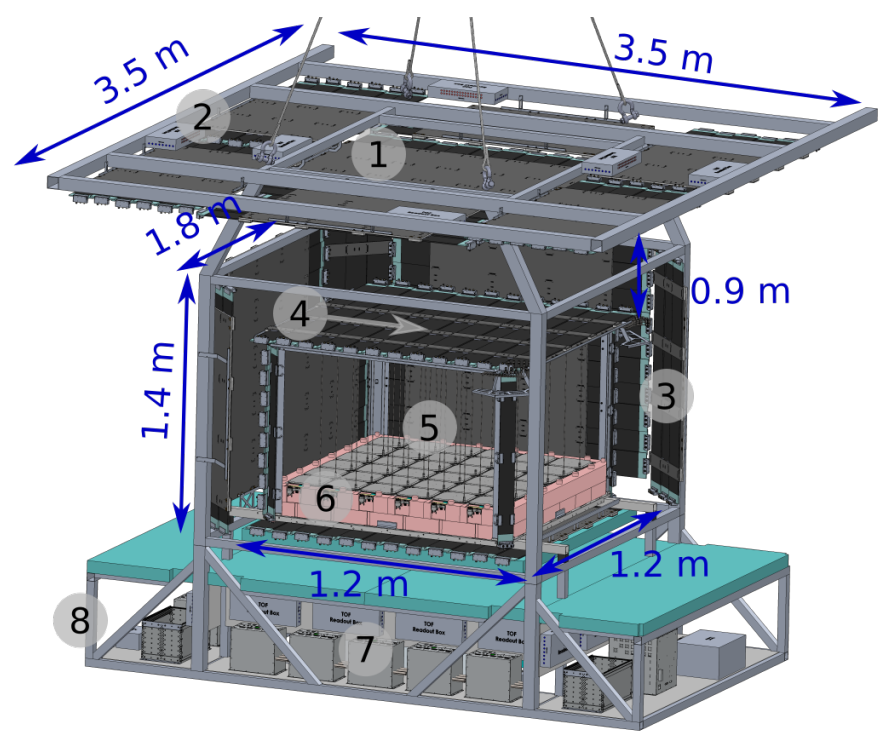

Figure 2: CAD model of the GAPS design with key dimensions labeled. (1) ToF umbrella (2) ToF electronics enclosure (3) ToF cortina (4) ToF cube (5) Si(Li) thermal system capillary tubing (6) Si(Li) detectors (7) ToF and $\mathrm{Si}(\mathrm{Li})$ electronics bay (8) gondola frame.

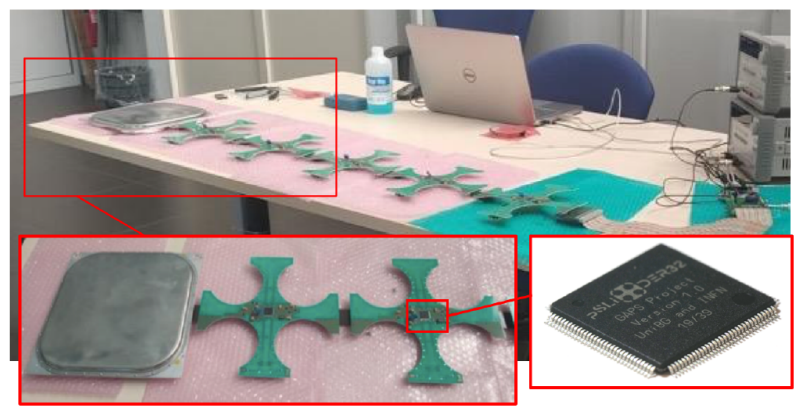

Figure 3: ASIC test stand in an end-to-end setup with a populated detector module and six fully-populated front-end boards in a daisy chained configuration.

compression that can be tuned with selectable peaking times from $0.3-1.8 \mu \mathrm{s}$. The ASIC digital outputs are routed into a back-end system that manages data acquisition and slow control processes. The first 32 prototype ASIC "pSLIDER32" testing has demonstrated all key functionality, and testing with the front-end board is on going, see Figure 3.

\subsection{Construction progress}

Despite the SARS-CoV2 pandemic of 2020, the construction of the $\mathrm{Si}(\mathrm{Li})$ subsystem continues. To date $1,116 \mathrm{Si}(\mathrm{Li})$ disks have been procured. Of that population hundreds have been passivated and calibrated (with 144 being made available for a scaled GAPS prototype detailed in a later section). About 3,456 strips have been calibrated and the population satisfying the resolution criterion has excellent performance: $\overline{\sigma_{\mathrm{FWHM}}}=3.1 \pm 0.5 \mathrm{keV}$. 

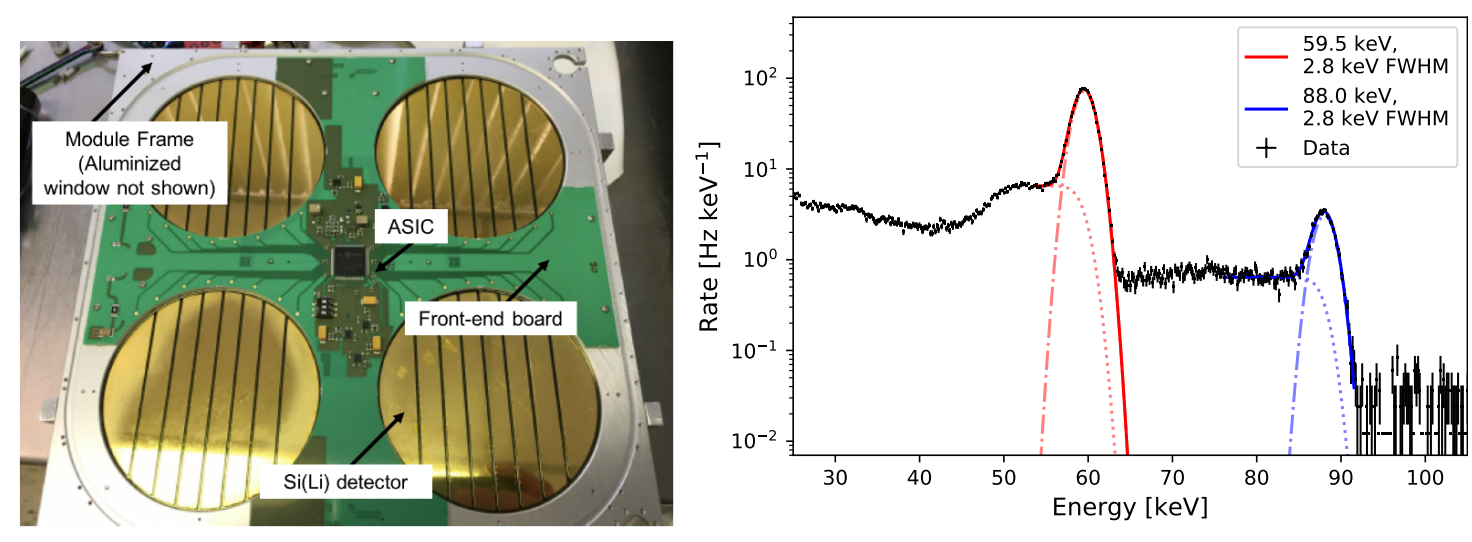

Figure 4: Left: Eight strip $\mathrm{Si}(\mathrm{Li})$ detector mounted in prototype module with example PCB that will be populated with custom ASIC. Right: Example calibration spectrum of one strip at $-35{ }^{\circ} \mathrm{C}$ [15].

\section{Plastic scintillator time-of-flight and trigger system}

The $\mathrm{Si}(\mathrm{Li})$ tracker target is surrounded by a two-layer (separation $\approx 90 \mathrm{~cm}$ ) plastic scintillator time-of-flight (TOF) system. The initial top layer, or "umbrella", restricts the acceptance to downward going primaries.

The second layer is an hermetic (in terms of particle tracks) "cube" encapsulating the $\mathrm{Si}(\mathrm{Li})$ tracker system. The main functions of the TOF are to: (1) calculate primary and secondary $\beta$, (2) provide additional tracking information for annihilation tracks and discriminate between in and outgoing particles and stopping time, (3) estimate primary composition, and (4) generating the trigger to read out the $\mathrm{Si}(\mathrm{Li})$ and TOF systems.

\subsection{Detector elements, geometry, and mechanical design}

Individual TOF counters are fabricated from Eljen EJ-200 plastic scintillator of thickness 6.35 $\mathrm{mm}$ and width $16 \mathrm{~cm}$. The long axis has four variants (previous dimensions being the same), with $1.8 \mathrm{~m}$ being used in the umbrella and cube, $1.56 \mathrm{~m}$ and $1.082 \mathrm{~m}$ lengths only in cube, and $1.72 \mathrm{~m}$ only in cortina. Since counters are overlapping in both layers, the faces will have close to $100 \%$ hermeticity. Additionally, tracks through overlapped regions (width of $1.09 \mathrm{~cm}$ or $3.07 \mathrm{~cm}$ ) provide enhanced position resolution along the narrow axis. Counters will be strapped to custom designed carbon fiber/foam sandwich panels and then mounted to the gondola frame. The overall structure is designed to withstand large impacts and meets NASA requirements for launch and recovery.

The machined scintillator (top/bottom as cast, sides and preamp end milled) is first wrapped with a smooth base layer of aluminum foil, and then overwrapped and sealed with opaque black plastic (Delta Blackout). A light weight U-channel assembly with attached preamp enclosure is secured directly to the scintillator using a cement-silicone-cement interface to withstand thermal expansion effects. 

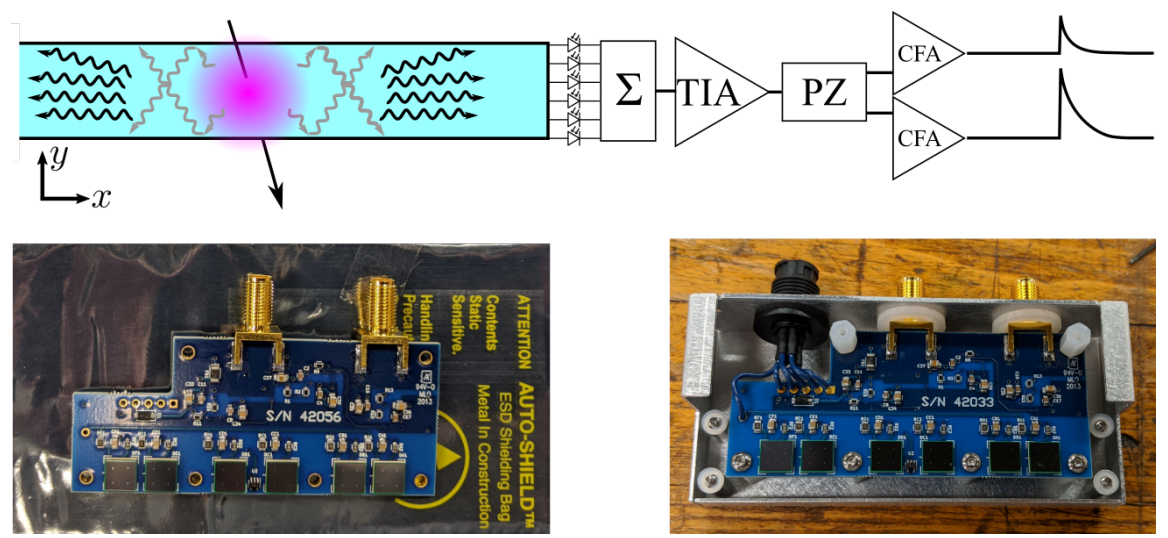

Figure 5: Top: block diagram of TOF signal generation. SiPM outputs are summed $(\Sigma)$, converted to voltage with transimpedance stage (TIA), shaped with pole-zero (PZ) and buffered by final current feedback stages (CFA) tuned for high or low gain output. Bottom: Bare preamp (left) and with enclosure assembly (right) prior to coupling to scintillator.

\subsection{Electronics}

The SiPM preamp uses a three stage cascaded design. (1) anode outputs from six SiPM (nominal gain of $G=4 \times 10^{6}$, maintained with temperature compensation via a custom power distribution board) are summed and passed to a (2) transimpedance amplifier whose output is shaped with pole zero cancellation which is subsequently split into a (3) current feedback stage with two outputs: a high gain timing channel with dynamic range of $\approx 25$ minimum ionizing particles (MIP) that is digitized, and a low gain trigger channel with dynamic range of $\approx 66$ MIP used for fast combinatorial logic. A diagram and photos are provided in Figure 5.

To achieve the best possible $\beta$ resolution given the $90 \mathrm{~cm}$ layer separation, high speed sampling is required (nominal value of $2 \mathrm{GS} / \mathrm{s}$ ). Because of its low power, high channel density, sample depth, and excellent bandwidth characteristics the DRS4 switched capacitor array (SCA) ASIC is used [18] in a custom readout board. The board provides in-situ amplitude and timing calibration systems which can be used to understand any environmental effects. Each board is connected to a gigabit LAN network for data transfer and remote access.

To process the large flux of cosmic rays at the top of atmosphere with a focus on identifying $\bar{p}$ and $\bar{d}$ candidate events, a fast, high bandwidth trigger system is being developed. The low gain outputs are connected to fast comparators that rapidly estimate a primary's deposited ionization energy $\mathrm{d} E / \mathrm{d} x$. The estimates for a local grouping of counters are processed, and a compressed data format of essential information is rapidly transmitted to a central system. Data from all local systems representing hit patterns are analyzed in real time, and when an interesting candidate is identified, a trigger is sent to the TOF readout system and $\mathrm{Si}(\mathrm{Li})$ tracker.

\subsection{Construction progress and current performance}

Extensive thermal and vacuum testing of the 4th generation SiPM preamps have validated their operation for the expected temperature and pressure ranges of flight. As of June, 2021 we have built and tested 60 preamps. Of these, 48 have been mounted to enclosures and coupled to sealed paddles. 

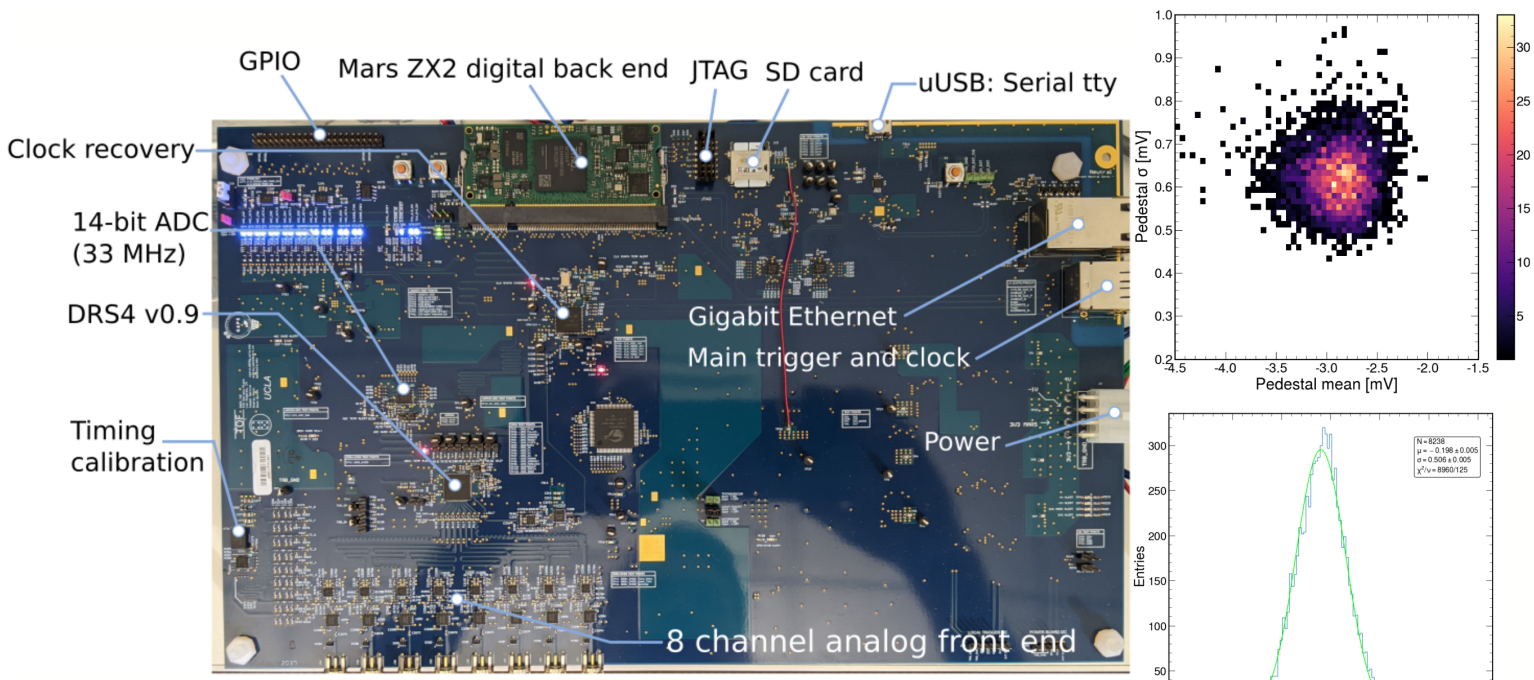

Figure 6: Left: Annotated photo of latest version DRS4 readout board in powered state. Right: Pedestal performance for channel 0 (top) and end-to-end timing performance for central muon data (bottom.)
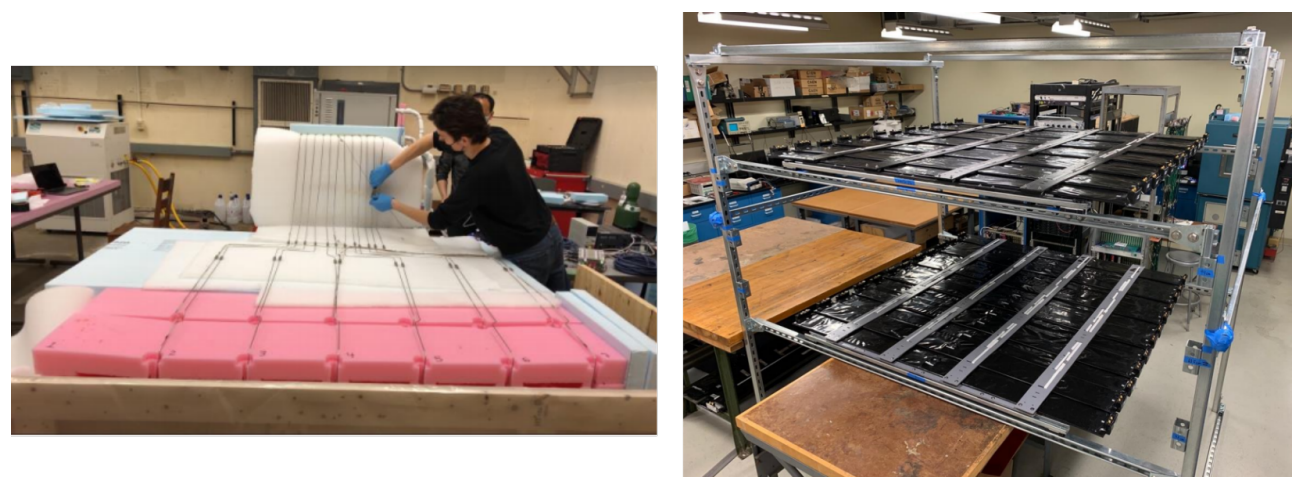

Figure 7: Left: a GAPS collaborator assembles the thermal system at Bates for further testing. Right: Completed construction of the two layer TOF system at UCLA, prior to electronic hookup.

The paddles are attached to a placeholder for the carbon fiber sandwich, but use the same strapping material and design as planned for the production version. Two layers have been completed and are mounted in a custom Unistrut framework to study downward going particles in the lab.

Currently four 2 nd generation read out boards have been produced and are being tested. The latest V2.4 version has been used to successfully digitize muon pulses from a production counter in a lab test bench. After performing amplitude calibration, but without timing calibration, the end-to-end paddle time difference is observed to be $\sigma\left(t_{a}-t_{b}\right) \approx 500$ ps with and low baseline noise, see Figure 6. This gives a current resolution of $\Delta t=\frac{\sigma\left(t_{a}-t_{b}\right)}{\sqrt{2}} \approx 358 \mathrm{~ns}$, compatible with the $\Delta t=500 \mathrm{ps}$ requirement. 


\section{Functional prototype}

To validate the performance of the integrated, flight model, system components, a scaled functional prototype (the GFP) is under construction at MIT's Bates lab during the winter of 2021. It will consist of the two TOF layers currently being tested, three layers of $\mathrm{Si}(\mathrm{Li})$ trackers in a $6 \times 2$ array of modules, and a scaled thermal system capable of reaching optimal temperatures.

The GFP will primarily study atmospheric muons and other minimum ionizing particles (MIPs). The construction is flexible and will permit the use of degraders to explore non-MIP particles with kinematics and $\mathrm{d} E / \mathrm{d} x$ similar to $T<0.25 \mathrm{GeV} / n \bar{p}$ and $\bar{d}$ we expect to observe in flight, and potentially muonic X-rays. In addition to a first pass at integrating the three independently developed systems, the GFP will provide an excellent test bed for preliminary data analysis and reconstruction tools and understanding the limitations of current hardware. An example of construction progress is shown in Figure 7.

\section{Conclusion}

The GAPS experiment is a highly sensitive cosmic ray antinucleus detector that will probe unexplored regions of dark matter parameter space and providing spectra in a new kinematic regime. This capability is enabled by a novel use of specialized $\mathrm{Si}(\mathrm{Li})$ technology and optimized TOF for the unique detector geometry. In this contribution we have summarized the design, implementation and current performance of the GAPS instrument systems while also reporting on the construction progress: hundreds of $\mathrm{Si}(\mathrm{Li})$ disks have been procured and calibrated along with a few dozen full assembled TOF counters. Subsystems continue to be locally tested while preparations are underway for a prototype integration at MIT Bates Lab later this winter.

\section{Acknowledgments}

This work is supported in the U.S. by NASA APRA grants (NNX17AB44G, NNX17AB45G, NNX17AB46G, and NNX17AB47G), in Japan by JAXA/ISAS Small Science Program FY2017, and in Italy by Istituto Nazionale di Fisica Nucleare (INFN) and by the Italian Space Agency through the ASI INFN agreement n. 2018-28-HH.0: "Partecipazione italiana al GAPS - General AntiParticle Spectrometer". R.A. Ong receives support from the UCL A Division of Physical Sciences. K. Perez receives support from the Heising-Simon Foundation and Alfred P. Sloan Foundation. F. Rogers is supported through the National Science Foundation Graduate Research Fellowship under grant 1122374. P. von Doetinchem receives support from the National Science Foundation under award PHY-1551980. H. Fuke receives support from JSPS KAKENHI grants JP26707015, JP17H01136, and JP19H05198. M. Kozai receives support from JSPS KAKENHI grant JP17K14313. S. Okazaki receives support from JSPS KAKENHI grant JP18K13928. Y. Shimizu receives support from Sumitomo Foundation grant.

\section{References}

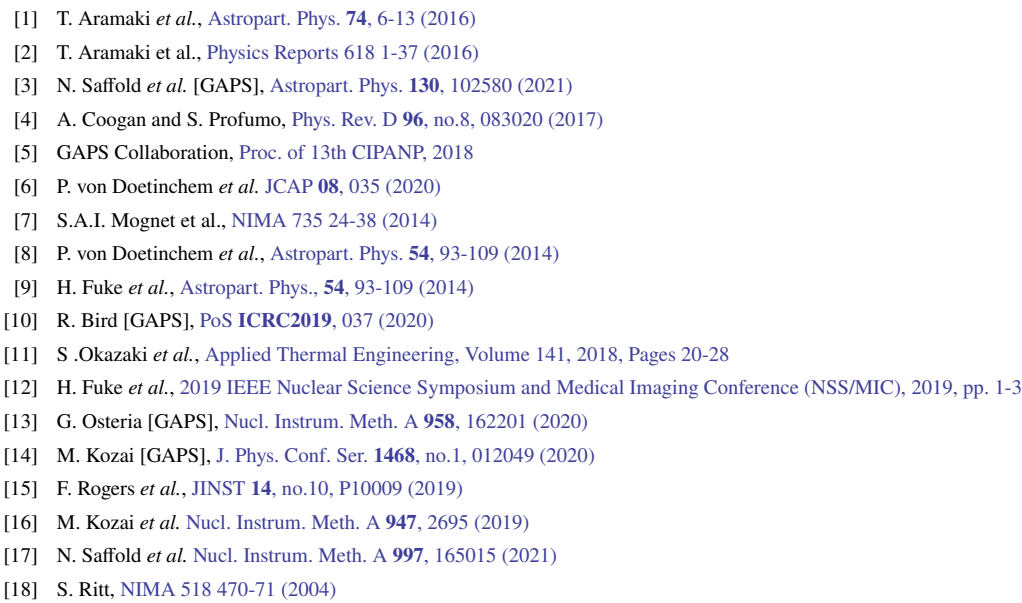




\section{Full Authors List: GAPS Collaboration}

T. Aramaki ${ }^{1}$, R. Bird ${ }^{2}$, M. Boezio ${ }^{3}$, S. E. Boggs ${ }^{4}$, V. Bonvicini ${ }^{3}$, D. Campana ${ }^{5}$, W. W. Craig ${ }^{6}$, E. Everson ${ }^{2}$, L. Fabris ${ }^{7}$, H. Fuke ${ }^{8}$, F. Gahbauer ${ }^{9}$, I. Garcia ${ }^{2}$, C. Gerrity ${ }^{10}$, C. J. Hailey ${ }^{9}$, T. Hayashi ${ }^{2}$, C. Kato ${ }^{11}$, A. Kawachi ${ }^{12}$, S. Kobayashi ${ }^{12}$, M. Kozai ${ }^{8}$, A. Lenni ${ }^{3,13}$, A. Lowell ${ }^{4}$, M. Manghisoni ${ }^{14,15}$, N. Marcelli ${ }^{16,17}$, M. Martucci ${ }^{16,17}$, S. I. Mognet ${ }^{18}$, K. Munakata ${ }^{11}$, R. Munini ${ }^{3}$, Y. Nakagami ${ }^{19}$, S. Okazaki ${ }^{8}$, J. Olson ${ }^{20}$, R. A. Ong ${ }^{2}$, G. Osteria ${ }^{5}$, K. Perez ${ }^{21}$, S. Quinn ${ }^{2}$, V. Re ${ }^{14,15}$, E. Riceputi ${ }^{14,15}$, B. Roach ${ }^{21}$, F. Rogers ${ }^{21}$, J. A. Ryan ${ }^{2}$, N. Saffold ${ }^{9}$, M. Saijo ${ }^{8}$, V. Scotti ${ }^{5,22}$, Y. Shimizu ${ }^{23}$, M. Sonzogni ${ }^{14,15}$, R. Sparvoli ${ }^{16,17}$, A. Stoessi ${ }^{10}$, K. Tokunaga ${ }^{8}$, E. Vannuccini2 ${ }^{24}$, P. von Doetinchem ${ }^{10}$, T. Wada $^{19}$, M. Xiao ${ }^{21}$, A. Yoshida ${ }^{19}$, T. Yoshida ${ }^{8}$, G. Zampa ${ }^{3}$, and J. Zweerink ${ }^{2}$ ${ }^{1}$ Northeastern University, 360 Huntington Avenue, Boston, MA 02115, USA ${ }^{2}$ University of California, Los Angeles, Los Angeles, CA 90095, USA ${ }^{3}$ INFN, Sezione di Trieste, I-34149 Trieste, Italy ${ }^{4}$ University of California, San Diego, La Jolla, CA 90037, USA ${ }^{5}$ INFN, Sezione di Napoli, I-80126 Naples, Italy ${ }^{6}$ Lawrence Livermore National Laboratory, Livermore, CA 94550, USA ${ }^{7}$ Oak Ridge National Laboratory, Oak Ridge, TN 37831, USA ${ }^{8}$ Institute of Space and Astronautical Science, Japan Aerospace Exploration Agency (ISAS/JAXA), Sagamihara, Kanagawa 252-5210, Japan ${ }^{9}$ Columbia University, New York, NY 10027, USA ${ }^{10}$ University of Hawaii at Manoa, Honolulu, HI 96822 USA ${ }^{11}$ Shinshu University, Matsumoto, Nagano 390-8621, Japan ${ }^{12}$ Tokai University, Hiratsuka, Kanagawa 259-1292, Japan ${ }^{13}$ Universitá di Trieste, I-34127 Trieste, Italy ${ }^{14}$ INFN, Sezione di Pavia, I-27100 Pavia, Italy ${ }^{15}$ Universitá di Bergamo, I-24044 Dalmine (BG), Italy ${ }^{16}$ INFN, Sezione di Rome "Tor Vergata", I-00133 Rome, Italy ${ }^{17}$ Universitá di Roma "Tor Vergata", I-00133 Rome, Italy ${ }^{18}$ Pennsylvania State University, University Park, PA 16802 USA ${ }^{19}$ Aoyama Gakuin University, Sagamihara, Kanagawa 252-5258, Japan ${ }^{20}$ Heliospace Corporation, Berkeley, CA 94710, USA ${ }^{21}$ Massachusetts Institute of Technology, Cambridge, MA 02139, USA ${ }^{22}$ Universitá di Napoli "Federico II", I-80138 Naples, Italy ${ }^{23}$ Kanagawa University, Yokohama, Kanagawa 221-8686, Japan ${ }^{24}$ INFN, Sezione di Firenze, I-50019 Sesto Fiorentino, Florence, Italy

Web version: gaps1.astro.ucla.edu/gaps/authors/ 